Abstract 406 Table 1 Clinicopathological features of patients with high grade endometrial cancer staged by lymphadenectomy

\begin{tabular}{|c|c|c|c|c|c|c|c|}
\hline & & $\begin{array}{r}\text { Total } \\
\mathrm{n}=251 \\
(\%) \\
\end{array}$ & $\begin{array}{r}\text { p53abn } \\
n=108 \\
(43.0 \%) \\
\end{array}$ & $\begin{array}{r}\text { POLEmut } \\
n=27 \\
(10.8 \%) \\
\end{array}$ & $\begin{array}{r}\text { MMRd } \\
n=78 \\
(31.1 \%) \\
\end{array}$ & $\begin{array}{r}\text { NSMP } \\
n=38 \\
(15.1 \%) \\
\end{array}$ & $P$ \\
\hline \multirow[t]{3}{*}{ Age, years } & & & & & & & 0.05 \\
\hline & $0-69$ & $152(60.6)$ & $56(51.9)$ & $23(85.2)$ & $50(64.1)$ & $23(60.5)$ & \\
\hline & $>70$ & $99(39.4)$ & $52(48.1)$ & $4(14.8)$ & $28(35.9)$ & $15(39.5)$ & \\
\hline \multirow[t]{5}{*}{ Histotype } & & & & & & & $<0.001$ \\
\hline & Endometrioid grade 3 & $107(42.6)$ & $25(23.1)$ & $16(59.3)$ & $48(61.5)$ & $18(47.4)$ & \\
\hline & Serous & $87(34.7)$ & $61(56.5)$ & $9(33.3)$ & $9(11.5)$ & $8(21.1)$ & \\
\hline & Clear cell & $51(21.3)$ & $18(16.7)$ & $2(7.4)$ & $19(24.4)$ & $12(31.6)$ & \\
\hline & Undifferentiated & $6(2.4)$ & $4(3.7)$ & $0(0)$ & $2(2.6)$ & $0(0)$ & \\
\hline \multirow[t]{7}{*}{ Stage } & & & & & & & 0.21 \\
\hline & IA & $115(45.8)$ & $53(49.1)$ & $15(55.6)$ & $34(43.6)$ & $13(34.2)$ & \\
\hline & IB & $57(22.7)$ & $21(19.4)$ & $10(37.0)$ & $16(20.5)$ & $10(26.3)$ & \\
\hline & II & $19(7.6)$ & $6(5.6)$ & $1(3.7)$ & $7(9.0)$ & $5(13.2)$ & \\
\hline & IIIA & $1(0.4)$ & $0(0)$ & $0(0)$ & $1(1.3)$ & $0(0)$ & \\
\hline & IIIB & $8(3.2)$ & $1(0.9)$ & $0(0)$ & $5(6.4)$ & $2(5.3)$ & \\
\hline & IIIC & $51(20.3)$ & $27(25.0)$ & $1(3.7)$ & $15(19.2)$ & $8(21.1)$ & \\
\hline \multicolumn{2}{|c|}{ Lymphovascular space invasion } & & & & & & 0.64 \\
\hline & Absent/focal & $210(83.7)$ & $89(82.4)$ & $25(92.6)$ & $63(80.7)$ & $33(86.8)$ & \\
\hline & Substantial & $35(13.9)$ & $15(13.9)$ & $2(7.4)$ & $14(17.9)$ & $4(10.5)$ & \\
\hline & Missing & $6(2.4)$ & $4(3.7)$ & $0(0)$ & $1(1.3)$ & $1(2.3)$ & \\
\hline \multicolumn{2}{|c|}{ Adjuvant treatment received } & & & & & & 0.61 \\
\hline & None & $178(70.9)$ & $77(71.3)$ & $22(81.5)$ & $53(67.9)$ & $26(68.4)$ & \\
\hline & Radiotherapy & $18(7.2)$ & $9(8.3)$ & $2(7.4)$ & $6(7.7)$ & $1(2.6)$ & \\
\hline & Chemotherapy & $49(19.5)$ & $20(18.5)$ & $2(7.4)$ & $18(23.1)$ & $9(23.7)$ & \\
\hline & Chemoradiotherapy & $6(2.4)$ & $2(1.9)$ & $1(1.3)$ & $1(1.3)$ & $2(5.3)$ & \\
\hline \multirow[t]{5}{*}{ ASA class } & & & & & & & 0.70 \\
\hline & 1 & $70(27.9)$ & $26(24.1)$ & $11(40.7)$ & $22(28.2)$ & $11(28.9)$ & \\
\hline & 2 & $163(64.9)$ & $73(67.6)$ & $13(48.1)$ & $52(66.7)$ & $25(65.8)$ & \\
\hline & 3 & $18(7.2)$ & $9(8.3)$ & $3(11.1)$ & $4(5.1)$ & $2(5.3)$ & \\
\hline & $4-5$ & $0(0)$ & $0(0)$ & $0(0)$ & $0(0)$ & $0(0)$ & \\
\hline
\end{tabular}

Result(s)* Molecular analysis was successful in 367 EC; 251 patients had undergone LND, see table 1. Median follow-up was 11 years (range 7.5-15.4). Multivariable analysis showed that molecular subgroup was a strong independent prognostic factor for recurrence: p53abn HR 3.88 (95\%CI 1.89-7.94, $p<0.001)$ and NSMP HR 4.80 (95\%CI 2.14-10.78, p<0.001) compared to MMRd. Figure $1 \mathrm{~A}$ shows time to recurrence among those patients staged by LND as IA-IB by molecular subgroup.

Among 264 patients without AT, 247 (94\%) had stage I-II disease and $17(6 \%)$ stage III. None of the patients with POLEmut EC $(\mathrm{n}=26,10 \%)$ had a recurrence (figure $1 \mathrm{~B})$. Multivariable analysis showed that the significant prognostic impact of molecular subgroup was independent of clinicopathological factors.

Conclusion* The molecular EC classification has strong prognostic value, independent of clinicopathological factors, also among patients staged by LND and those without AT. This implies that the unfavourable prognosis of p53abn EC is not caused by undetected lymph node metastasis. POLEmut EC is inherently associated with an excellent prognosis even in the absence of adjuvant treatment.

\section{PERFORMANCE OF DNA METHYLATION ANALYSIS IN URINE, CERVICOVAGINAL SELF-SAMPLES AND CERVICAL SCRAPES FOR ENDOMETRIAL CANCER DETECTION}

${ }^{1 ; 2} \mathrm{R}$ Van den Helder*, ${ }^{2} \mathrm{BMM}$ Wever, ${ }^{2} \mathrm{AP}$ Van Splunter, ${ }^{3} \mathrm{CH}$ Mom, ${ }^{3} \mathrm{~J}$ Kasius, ${ }^{2}$ RDM Steenbergen, ${ }^{1} \mathrm{~N}$ Van Trommel, ${ }^{2} \mathrm{MCG}$ Bleeker. ${ }^{7}$ The Netherlands Cancer Institute (NKI), Department of Gynecologic Oncology, Amsterdam, Netherlands; ${ }^{2}$ Amsterdam University Medical Center (UMC), Vrije Universiteit Amsterdam, Department of Pathology, Amsterdam, Netherlands; ${ }^{3}$ Amsterdam UMC, locatie AMC, Department of Gynecologic Oncology, Amsterdam, Netherlands

10.1136/ijgc-2021-ESGO.139 
Introduction/Background* Urine may offer an alternative sample type for gynecologic cancer detection1, which is easily accessible and allows self-sampling at home. DNA methylation is an emerging biomarker for early cancer detection, and the feasibility of endometrial cancer detection in urine using DNA methylation analysis has recently been reported2. This study aimed to determine the performance of DNA methylation analysis in urine for endometrial cancer detection, and to make a comparison to paired cervicovaginal self-samples and cervical scrapes.

Methodology From 110 women diagnosed with endometrial cancer, paired urine samples, cervicovaginal self-samples and cervical scrapes were collected as well as samples from agematched healthy female controls. All samples were tested for six DNA methylation markers. Differences in DNA methylation levels between patients and controls were compared using the non-parametric Mann-Whitney U-test, and the performance was quantified by the area under the receiver operating characteristic (ROC) curve (AUCs) and logistic regression. Correlation of DNA methylation markers within paired sample types was determined using the Spearman correlation coefficients.

Result(s)* In urine, self-samples and cervical scrapes, all six DNA methylation markers showed increased methylation levels in patients as compared to controls. Analyses amongst the paired sample types showed a good correlation between the test results of the DNA methylation markers.

Conclusion* This study demonstrates that testing for DNA methylation markers in urine may provide an easy and accurate alternative method for the detection of endometrial cancer. Potential applications of this diagnostic approach include the screening of asymptomatic women, triaging women with (postmenopausal) bleeding symptoms, and monitoring women with increased endometrial cancer risk.

\section{LONG-TERM OUTCOME IN ENDOMETRIAL CANCER PATIENTS AFTER ROBOT-ASSISTED LAPAROSCOPIC SURGERY WITH SENTINEL LYMPH NODE MAPPING}

\begin{abstract}
${ }^{1 ; 2} \mathrm{NJ}$ Nordskar*, ${ }^{2} \mathrm{~B}$ Hagen, ${ }^{2} \mathrm{~S}$ Tingulstad, ${ }^{3} \mathrm{EV}$ Vesterfjell, ${ }^{4} \varnothing$ Salvesen, ${ }^{1 ; 2} \mathrm{G}$ Aune. ${ }^{1}$ Norwegian University of Science and Technology, Department of Clinical and Molecular Medicine, Trondheim, Norway; ${ }^{2}$ St Olavs hospital, Trondheim University hospital, Department of Gynecologic Oncology, Trondheim, Norway; ${ }^{3}$ St Olavs hospital, Trondheim University hospital, Department of Pathology, Trondheim, Norway; ${ }^{4}$ Unit of Applied Clinical Research, Department of Public Health and Nursing, Trondheim, Norway
\end{abstract}

\subsection{6/ijgc-2021-ESGO.140}

Introduction/Background* The aim was to provide long-term outcome data in endometrial cancer patients undergoing robot-assisted laparoscopic surgery and sentinel lymph node (SLN) mapping.

Methodology Retrospective cohort study of 108 patients with primary endometrial cancer who underwent robot-assisted laparoscopic surgery and sentinel lymph node mapping using the Memorial Sloan Kettering Cancer Center algorithm with nearinfrared fluorescence detection of indocyanine green for endometrial cancer from November 20th 2012 to January 1st 2016 at St. Olavs hospital in Norway. The primary endpoint was recurrence-free survival. Secondary endpoints were overall survival and treatment complications.

Result(s)* After a median follow up of 75 months (range 61$98)$, five $(4.6 \%)$ patients had recurred and three patients had died from the disease. The 5 -year recurrence-free survival was 95.4\% (95\% CI, 91.5 - 99.3). The 5-year disease specific survival was $97.2 \%$ (95\% CI, 94.1 - 100.3). Four of the patients with recurrent disease had lymph node metastasis at diagnosis. The 5-year overall survival was 92.6\% (95\% CI, 87.7 - 97.5). Peripheral neuropathy after chemotherapy was the most common complication (9.3\%), followed by lower limb edema $(2 \%)$ and postoperative hernia (2\%).

Conclusion* The present study demonstrated excellent oncologic outcome and few treatment complications in patients treated according to the SLN algorithm more than five years after diagnosis.

\section{BIOENDOCAR: OMICS APPROACHES FOR DIAGNOSIS AND PROGNOSIS OF ENDOMETRIAL CANCER}

${ }^{1} \mathrm{E}$ Hafner*, ${ }^{2} \zeta$ S Smrkolj, ${ }^{3} \mathrm{~A}$ Romano, ${ }^{3} \mathrm{HMJ}$ Werner, ${ }^{4} \mathrm{~A}$ Semczuk, ${ }^{4} S$ Wawrysiuk, ${ }^{4} \mathrm{~A}$ Adamiak-Godlewska, ${ }^{5 ; 6} \mathrm{D}$ Fishman, ${ }^{5 ; 6}$ J Vilo, ${ }^{7} \mathrm{C}$ Lowy, ${ }^{7} \mathrm{C}$ Schröder, ${ }^{8} \mathrm{~J} \mathrm{Tokarz,}$ ${ }^{9} \mathrm{~V}$ Weinberger, ${ }^{9} \mathrm{M}$ Bednařiková, ${ }^{9} \mathrm{P}$ Vinklerova, ${ }^{10} \mathrm{~S}$ Ferrero, ${ }^{10} \mathrm{~F}$ Barra, ${ }^{11} \mathrm{I}$ Takač, ${ }^{11} \mathrm{~J} \mathrm{Knez}$, ${ }^{1} T$ Lanišnik Rižner. 'Institute of Biochemistry and Molecular Genetics, Faculty of Medicine, University of Ljubljana, Ljubljana, Slovenia; ${ }^{2}$ University Medical Centre Ljubljana, Department of Obstetrics and Gynecology, Ljubljana, Slovenia; ${ }^{3}$ Department of Obstetrics and Gynecology, GROW, School for Oncology and Developmental Biology, Maastricht University Medical Centre, Maastricht, Netherlands; ${ }^{4}$ Department of Gynecology, Lublin Medical University, Lublin, Poland; ${ }^{5}$ Institute of Computer Science, University of Tartu, Tartu, Estonia; ${ }^{6}$ Quretec Ltd., Tartu, Estonia; ${ }^{7}$ Sciomics GmbH, Heidelberg, Germany; ${ }^{8}$ Institute for Diabetes and Cancer, Helmholtz Zentrum, Munich, Germany; ${ }^{9}$ Department of Gynecology and Obstetrics, University Hospital Brno, Brno, Czech Republic; ${ }^{10}$ Academic Unit of Obstetrics and Gynecology, IRCCS Ospedale Policlinico San Martino, Genoa, Italy; ${ }^{11}$ University Medical Centre Maribor, Division of Gynecology and Perinatology, Maribor, Slovenia

\subsection{6/ijgc-2021-ESGO.141}

Introduction/Background* Endometrial cancer is the second most common carcinoma of the female genital tract globally, and its incidence is still increasing. Optimal treatment of EC depends on early diagnostics and pre-operative stratification to appropriately select the extent of surgery and to plan further therapeutic approach. Current diagnosis and treatment of EC patients is guided by histopathological and surgical findings since there are no accurate non-invasive diagnostic or prognostic methods available. The lack of non-invasive diagnostic and prognostic biomarkers of EC is addressed in the current clinical study titled 'Biomarkers for Diagnosis and Prognosis of Endometrial Carcinoma' (NCT03553589).

Methodology Patient recruitment takes place at six medical centers (University Medical Centre Ljubljana, Slovenia; University Medical Centre Maribor, Slovenia; Maastricht University Medical Centre, The Netherlands; Lublin Medical University, Poland; University Hospital Brno, Czech Republic, University of Genoa, Italy).

Plasma samples from women with diagnosed EC and controls will be examined using non-targeted and targeted metabolomics and targeted proteomics approaches. Combined blood metabolome (>850 metabolites), proteome (>900 proteins), clinical and epidemiological data will be analyzed in order to construct diagnostic/prognostic algorithms for early diagnosis of EC and to identify patients with low/high risk for cancer progression and recurrence.

BioEndoCar consortium has defined inclusion/exclusion criteria and a strict standard operating procedure for sample collection, processing and storage that is followed in all medical centers. 